\title{
Charm meson scattering cross sections by pion and rho meson
}

\author{
Ziwei Lin, T. G. Di, and C. M. Ko \\ Cyclotron Institute and Physics Department, Texas A\&M University, College Station, Texas \\ 7r7843-3366
}

\begin{abstract}
Using the local flavor SU(4) gauge invariance in the limit of vanishing vector meson masses, we extend our previous study of charm meson scattering cross sections by pion and rho meson, which is based only on the pseudoscalarpseudoscalar-vector meson couplings, to include also contributions from the couplings among three vector mesons and among four particles. We find that diagrams with light meson exchanges usually dominate the cross sections. For the processes considered previously, the additional interactions lead only to diagrams involving charm meson exchanges and contact interactions, and the cross sections for these processes are thus not much affected. Nevertheless, these additional interactions introduce new processes with light meson exchanges and increase significantly the total scattering cross sections of charm mesons by pion and rho meson.
\end{abstract}

PACS number(s): 25.75.-q, 13.75.Lb, 14.40.Lb

\section{INTRODUCTION}

Since charm quarks may lose appreciable energies in a quark-gluon plasma via gluon radiations, study of the charm meson spectrum in heavy ion collisions is expected to provide useful information on the properties of the quark-gluon plasma formed in these collisions [1.22]. However, charm mesons may interact strongly with hadrons during later stage of heavy ion collisions, and this may also lead to changes in their final spectrum. To use charm mesons as a probe of the properties of the initial quark-gluon plasma thus requires the understanding of their interactions with hadrons. In a previous study [3], we have evaluated the charm meson scattering cross sections with pion and rho meson in a simple hadronic model that includes only the pseudoscalar-pseudoscalar-vector (PPV) meson interactions. In that study, we have also neglected the exchange of charm mesons as it is expected to be unimportant due to the large charm meson mass. Including form factors at the interaction vertices, we have obtained a thermally averaged total cross section of about $10 \mathrm{mb}$ in the temperature range of interest. In a schematic model for the dynamics of heavy ion collisions, we have found that the inverse slope of the charm meson transverse momentum spectrum is significantly enhanced by their scatterings in the hadronic matter. As a result, the invariant mass spectrum of the dileptons from the decay of charm meson pairs is expected to be modified, which has been suggested as a possible explanation for the observed enhancement of intermediate-mass dileptons in heavy ion collisions at SPS energies [4]. 
In Ref. [5], Matinyan and Müller have used a similar hadronic Lagrangian to evaluate the cross sections of charmonium absorption in hadronic matter. In contrast with the charm meson scattering cross sections, they have obtained very small charmonium absorption cross sections. The model has been extended in Refs. [6,7] by using the local flavor SU(4) gauge invariance to include also interactions among three vector mesons and among four particles. Because of these additional interactions, the charmonium absorption cross sections are increased by an order-of-magnitude. In this paper, we shall use this extended hadronic Lagrangian to study the charm meson scattering cross sections by pion and rho meson.

The paper is organized as follows. In Sec. ПIA, we introduce the hadronic Lagrangian based on the local flavor SU(4) gauge symmetry. The interaction Lagrangians that are relevant to charm meson scattering with pion and rho meson are then given in Sec. IIB. We also derive in this section the scattering amplitudes for these processes and give their explicit expressions in Appendix A. Constraints on the scattering amplitudes as a result of the conservation of SU(4) flavor current are then discussed in Sec. IIT, and an example is shown in detail in Appendix B. After addressing in Sec. IID the problem of on-shell divergence in some of the amplitudes, we fix the coupling constants in Sec. [IE and introduce in Sec. IIF the form factors at interaction vertices. Numerical results for the charm meson scattering cross sections are presented in Sec. [II]. In Sec. IV], we compare our results with previous ones obtained using the PPV coupling and including only diagrams with light meson exchanges. Finally, a summary is given in Sec. D.

\section{CHARM MESON INTERACTIONS WITH HADRONS}

\section{A. hadronic Lagrangian with SU(4) Symmetry}

We have previously introduced a hadronic Lagrangian with SU(4) symmetry for studying the charmonium absorption cross sections by hadrons [7]. It starts from the free Lagrangian for pseudoscalar and vector mesons,

$$
\mathcal{L}_{0}=\operatorname{Tr}\left(\partial_{\mu} P^{\dagger} \partial^{\mu} P\right)-\frac{1}{2} \operatorname{Tr}\left(F_{\mu \nu}^{\dagger} F^{\mu \nu}\right),
$$

where $F_{\mu \nu}=\partial_{\mu} V_{\nu}-\partial_{\nu} V_{\mu}$, and $P$ and $V$ denote, respectively, the $4 \times 4$ pseudoscalar and vector meson matrices in $\mathrm{SU}(4)$ [3:0]. Introducing the minimal substitution,

$$
\begin{aligned}
\partial_{\mu} P & \rightarrow \mathcal{D}_{\mu} P=\partial_{\mu} P-\frac{i g}{2}\left[V_{\mu}, P\right], \\
F_{\mu \nu} & \rightarrow \partial_{\mu} V_{\nu}-\partial_{\nu} V_{\mu}-\frac{i g}{2}\left[V_{\mu}, V_{\nu}\right],
\end{aligned}
$$

leads to the following Lagrangian for the interacting hadrons:

$$
\begin{aligned}
\mathcal{L} & =\mathcal{L}_{0}+i g \operatorname{Tr}\left(\partial^{\mu} P\left[P, V_{\mu}\right]\right)-\frac{g^{2}}{4} \operatorname{Tr}\left(\left[P, V_{\mu}\right]^{2}\right) \\
& +i g \operatorname{Tr}\left(\partial^{\mu} V^{\nu}\left[V_{\mu}, V_{\nu}\right]\right)+\frac{g^{2}}{8} \operatorname{Tr}\left(\left[V_{\mu}, V_{\nu}\right]^{2}\right) .
\end{aligned}
$$

Since hadron masses explicitly break the SU(4) symmetry, mass terms based on the experimentally determined values are added to Eq. (四). 


\section{B. scattering amplitudes}

The above Lagrangian yields the following processes for charm meson scattering by $\pi$ and $\rho$ mesons,

$$
\pi D \leftrightarrow \rho D^{*}, \pi D \rightarrow \pi D, \pi D^{*} \rightarrow \pi D^{*}, \pi D^{*} \leftrightarrow \rho D, \rho D \rightarrow \rho D, \rho D^{*} \rightarrow \rho D^{*} .
$$

There are also similar processes for anti-charm mesons. Fig. 1 shows the diagrams for the eight processes in Eq. (5).
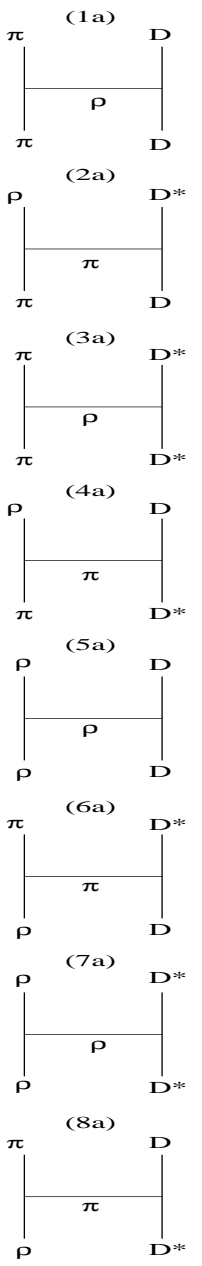
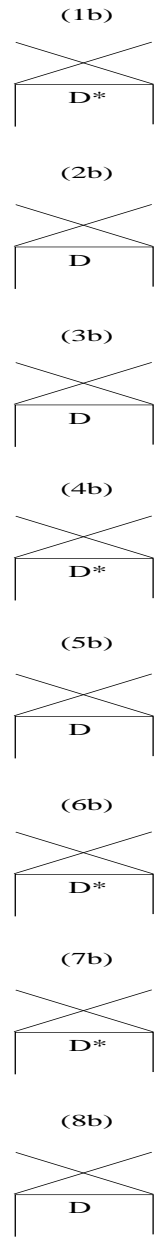
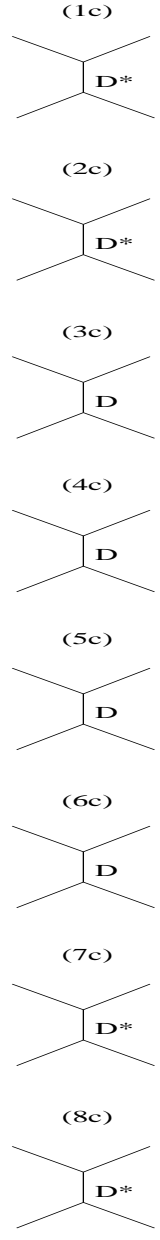
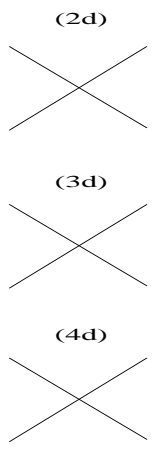

(5d)

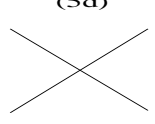

(6d)

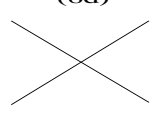

(7d)

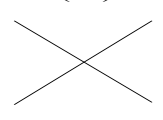

(8d)

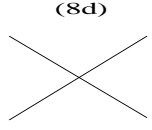

FIG. 1. Diagrams for charm meson scattering by pion and rho meson. The numbers denote different processes, and the Roman letters label the different amplitudes in a given process.

Expanding the Lagrangian in Eq. (4) using the $4 \times 4$ matrices for $P$ and $V$, we obtain the following interaction Lagrangians that are relevant to charm meson scattering:

$$
\begin{aligned}
\mathcal{L}_{\rho \pi \pi} & =g_{\rho \pi \pi} \vec{\rho}^{\mu} \cdot\left(\vec{\pi} \times \partial_{\mu} \vec{\pi}\right) \\
\mathcal{L}_{\rho \rho \rho} & =-g_{\rho \rho \rho} \partial_{\mu} \overrightarrow{\rho_{\nu}} \cdot\left(\overrightarrow{\rho^{\mu}} \times \overrightarrow{\rho^{\nu}}\right) \\
\mathcal{L}_{\pi D D^{*}} & =i g_{\pi D D^{*}} D^{* \mu} \vec{\tau} \cdot\left(\bar{D} \partial_{\mu} \vec{\pi}-\partial_{\mu} \vec{D} \vec{\pi}\right)+\text { H.c. }
\end{aligned}
$$




$$
\begin{aligned}
\mathcal{L}_{\rho D D} & =i g_{\rho D D}\left(D \vec{\tau} \partial_{\mu} \bar{D}-\partial_{\mu} D \vec{\tau} \bar{D}\right) \cdot \vec{\rho}^{\mu} \\
\mathcal{L}_{\rho D^{*} D^{*}} & =i g_{\rho D^{*} D^{*}}\left[\left(\partial_{\mu} D^{* \nu} \vec{\tau} \bar{D}_{\nu}^{*}-D^{* \nu} \vec{\tau} \partial_{\mu} \bar{D}_{\nu}^{*}\right) \cdot \vec{\rho}^{\mu}\right. \\
& \left.+\left(D^{* \nu} \vec{\tau} \cdot \partial_{\mu} \vec{\rho}_{\nu}-\partial_{\mu} D^{* \nu} \vec{\tau} \cdot \vec{\rho}_{\nu}\right) \bar{D}^{* \mu}+D^{* \mu}\left(\vec{\tau} \cdot \vec{\rho}^{\nu} \partial_{\mu} \bar{D}_{\nu}^{*}-\vec{\tau} \cdot \partial_{\mu} \vec{\rho}^{\nu} \bar{D}_{\nu}^{*}\right)\right], \\
\mathcal{L}_{\pi \rho D D^{*}} & =-g_{\pi \rho D D^{*}} D^{* \mu}\left(2 \vec{\tau} \cdot \vec{\pi} \vec{\tau} \cdot \overrightarrow{\rho_{\mu}}-\vec{\tau} \cdot \overrightarrow{\rho_{\mu}} \vec{\tau} \cdot \vec{\pi}\right) \bar{D}+\text { H.c. } \\
\mathcal{L}_{\pi \pi D^{*} D^{*}} & =g_{\pi \pi D^{*} D^{*}}\left(\frac{\vec{\pi} \cdot \vec{\pi}}{2}\right) D^{* \mu} \bar{D}_{\mu}^{*}, \\
\mathcal{L}_{\rho \rho D D} & =g_{\rho \rho D D}\left(\frac{\overrightarrow{\rho_{\mu}} \cdot \overrightarrow{\rho^{\mu}}}{2}\right) D \bar{D}, \\
\mathcal{L}_{\rho \rho D^{*} D^{*}} & =g_{\rho \rho D^{*} D^{*}} D^{* \mu}\left(2 \vec{\tau} \cdot \overrightarrow{\rho_{\nu}} \vec{\tau} \cdot \overrightarrow{\rho_{\mu}}-\vec{\tau} \cdot \overrightarrow{\rho_{\mu}} \vec{\tau} \cdot \overrightarrow{\rho_{\nu}}-\overrightarrow{\rho_{\gamma}} \cdot \overrightarrow{\rho^{\gamma}} g_{\mu \nu}\right) \bar{D}^{* \nu} .
\end{aligned}
$$

In the above, $\vec{\tau}$ are Pauli matrices; $\vec{\pi}$ and $\vec{\rho}$ denote the pion and rho meson isospin triplets, respectively; while $D$ and $D^{*}$ denote the pseudoscalar and vector charm meson isospin doublets, respectively.

Using the above interacting Lagrangians, we have derived the amplitudes for all diagrams in Fig. I, and they are given in Appendix A. In general, the amplitude for a process $n$, before summing and averaging over external spins and isospins, is given by the coherent sum of all individual amplitudes that contributing to the process, i.e.,

$$
\mathcal{M}_{n}=\left(\sum_{i} \mathcal{M}_{n i}^{\lambda_{k} \cdots \lambda_{l}}\right) \epsilon_{k \lambda_{k}} \cdots \epsilon_{l \lambda_{l}} \equiv \mathcal{M}_{n}^{\lambda_{k} \cdots \lambda_{l}} \epsilon_{k \lambda_{k}} \cdots \epsilon_{l \lambda_{l}}
$$

where $i$ runs through $a, b, c$ for process 1 and $a, b, c, d$ for all other processes, and $\epsilon_{j \lambda_{j}}$ denotes the polarization vector of external vector meson $j$.

\section{C. current conservation}

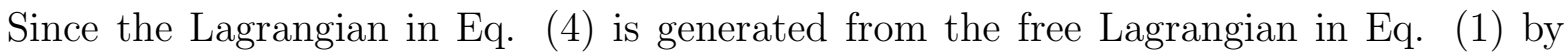
the minimal substitution, it is invariant under the local flavor $\mathrm{SU}(4)$ gauge transformation, thus it is also invariant under the global flavor SU(4) gauge transformation. This invariance remains valid after including degenerate pseudoscalar and degenerate vector meson mass terms, leading to the conservation of a $\mathrm{SU}(4)$ flavor current. As a result, the scattering amplitude for any process satisfies the following condition:

$$
\mathcal{M}_{n}^{\lambda_{k} \ldots \lambda_{l}} p_{j \lambda_{j}}=0,
$$

where $p_{j \lambda_{j}}$ is the momentum of external vector meson $j$. As an example, we show explicitly in Appendix B that the condition $\mathcal{M}_{2}^{\lambda \omega} p_{4 \omega}=0$ is indeed satisfied by the amplitude for process $2, \pi D \rightarrow \rho D^{*}$.

\section{D. on-shell divergence}

The amplitudes for diagrams 3b, 4a, and $6 \mathrm{a}$ become singular when the exchanged mesons are on-shell. Since the on-shell process describes a two-step process, their contribution needs 
to be subtracted from the cross section. Several methods have been proposed to treat such a singularity [8]. Since we are interested in charm meson scattering in hadronic matter, the exchanged meson is expected to acquire an imaginary self-energy due to collisional broadening. The one-step process then corresponds to keeping only the real part of the propagator for the exchanged meson. However, a consistent evaluation of this effect also requires the inclusion of vertex corrections due to the medium, which has not been carried out even for light meson scattering in hadronic matter. We thus follow Ref. [3] by adding an imaginary part of $50 \mathrm{MeV}$ to the self-energy of the exchanged meson in the above three diagrams. Since the width of $D^{*}$ in vacuum is very small (about $44 \mathrm{KeV}$ ) [9], the amplitude

for diagram 1c can also be very large when the center-of-mass energy of the initial pion and charm meson is close to the $D^{*}$ mass. We thus also add an imaginary part of $50 \mathrm{MeV}$ to the self-energy of the $D^{*}$ meson in diagram 1c. In Sec. III we shall show that thermal averages of these cross sections do not change much for values of imaginary self-energy between 5 and $500 \mathrm{MeV}$.

\section{E. coupling constants}

For the coupling constants in the interaction Lagrangians, we shall use empirical values if they are available, i.e. $g_{\rho \pi \pi}=6.1$ [10], $g_{\pi D D^{*}}=4.4$ [9]. $g_{\rho D D}=g_{\rho D^{*} D^{*}}=2.52$ [5], [7]. Since there is little empirical information on other coupling constants, we use the $\mathrm{SU}(4)$ relations to determine their values, i.e.,

$g_{\rho \rho \rho}=g_{\rho \pi \pi}, g_{\pi \rho D D^{*}}=g_{\pi D D^{*}} g_{\rho D D}, g_{\pi \pi D^{*} D^{*}}=2 g_{\pi D D^{*}}^{2}, g_{\rho \rho D D}=2 g_{\rho D D}^{2}, g_{\rho \rho D^{*} D^{*}}=g_{\rho D^{*} D^{*}}^{2}$.

We note that the SU(4) symmetry gives the following relations among couplings constants:

$$
\begin{aligned}
& \frac{g_{\rho \pi \pi}}{2}(3.0)=g_{\pi D D^{*}}(4.4)=g_{\rho D D}(2.5)=\frac{g_{\rho \rho \rho}}{2}=g_{\rho D^{*} D^{*}}=\frac{g}{4} \\
& g_{\pi \rho D D^{*}}=\frac{g_{\pi \pi D^{*} D^{*}}}{2}=\frac{g_{\rho \rho D D}}{2}=g_{\rho \rho D^{*} D^{*}}=\frac{g^{2}}{16} .
\end{aligned}
$$

The empirical values given in the parentheses are seen to agree reasonably with those predicted by the SU(4) symmetry.

\section{F. form factors}

Because of the finite size of hadrons, form factors are needed at interaction vertices. In the present study, we take them to be the usual mono-pole form for vertices in the $t$ and $u$ channel processes, i.e.,

$$
f_{3}(t \text { or } u)=\frac{\Lambda^{2}}{\Lambda^{2}+\mathbf{q}^{2}},
$$

where $\Lambda$ is a cutoff parameter, and $\mathbf{q}^{2}$ is the squared three momentum transfer in the centerof-mass frame, given by $\left(\mathbf{p}_{\mathbf{1}}-\mathbf{p}_{\mathbf{3}}\right)_{\mathrm{cm}}^{2}$ and $\left(\mathbf{p}_{\mathbf{1}}-\mathbf{p}_{\mathbf{4}}\right)_{\mathrm{cm}}^{2}$, respectively, for the $t$ and $u$ channel 
processes. These form factors are different from that used in Ref. [3], where it is given by $f(t)=\left(\Lambda^{2}-m^{2}\right) /\left(\Lambda^{2}-t\right)$, since the latter is not suitable for diagrams involving the charmed meson exchange that has a large invariant four momentum transfer $t$.

As in Ref. [11], form factors at $s$ channel vertices are taken as

$$
f_{3}(s)=\frac{\Lambda^{2}}{\Lambda^{2}+\mathbf{k}^{2}}
$$

with $\mathbf{k}$ denoting the three momentum of either the incoming or outgoing particles in the center-of-mass, i.e., $\mathbf{k}^{2}=p_{i, \mathrm{~cm}}^{2}$ or $p_{f, \mathrm{~cm}}^{2}$.

After introducing the form factors at three-point vertices, the form factors at four-point vertices can in principle be determined by requiring the total amplitude for a given process satisfies the current conservation condition of Eq. (8) [12,13. Since the uncertainty of form factors involving charm mesons is already large for three-point vertices and the gauge invariance is not valid once we use empirical vector meson masses, we choose not to follow this more involved approach. Instead, we simply take the form factors at four-point vertices to be

$$
f_{4}=\left(\frac{\Lambda^{2}}{\Lambda^{2}+\bar{q}^{2}}\right)^{2}
$$

where $\overline{\mathbf{q}^{2}}$ is the average value of the squared three momenta in the form factors for the $s, t$, and $u$ channels, i.e.,

$$
\overline{\mathbf{q}^{2}}=\frac{5}{6}\left(p_{i, \mathrm{~cm}}^{2}+p_{f, \mathrm{~cm}}^{2}\right)
$$

Since there is no empirical information on form factors involving charm mesons, we shall use for simplicity the same value for all cutoff parameters and choose $\Lambda$ as either 1 or $2 \mathrm{GeV}$ to study the uncertainties of our results due to form factors.

\section{G. thermally averaged cross sections}

For charm meson scattering in hadronic matter, it is useful to study the thermal average of their cross sections. For a hadronic matter at temperature $T$, this is given by

$$
\langle\sigma v\rangle=\frac{\int_{z_{0}}^{\infty} d z\left[z^{2}-\left(\alpha_{1}+\alpha_{2}\right)^{2}\right]\left[z^{2}-\left(\alpha_{1}-\alpha_{2}\right)^{2}\right] K_{1}(z) \sigma\left(s=z^{2} \mathrm{~T}^{2}\right)}{4 \alpha_{1}^{2} K_{2}\left(\alpha_{1}\right) \alpha_{2}^{2} K_{2}\left(\alpha_{2}\right)}
$$

where $\alpha_{i}=m_{i} / \mathrm{T}, z_{0}=\max \left(\alpha_{1}+\alpha_{2}, \alpha_{3}+\alpha_{4}\right), K_{n}$ 's are modified Bessel functions, and $v$ is the relative velocity of initial-state particles in their collinear frame [14].

\section{NUMERICAL RESULTS}

We first consider the case without form factors at interaction vertices, i.e., $\Lambda=\infty$. In Fig. 2, the solid lines show the energy dependence of the total charm meson scattering cross section for a given initial state, i.e., processes 1 and 2 for $\pi D$ scattering, processes 3 and 


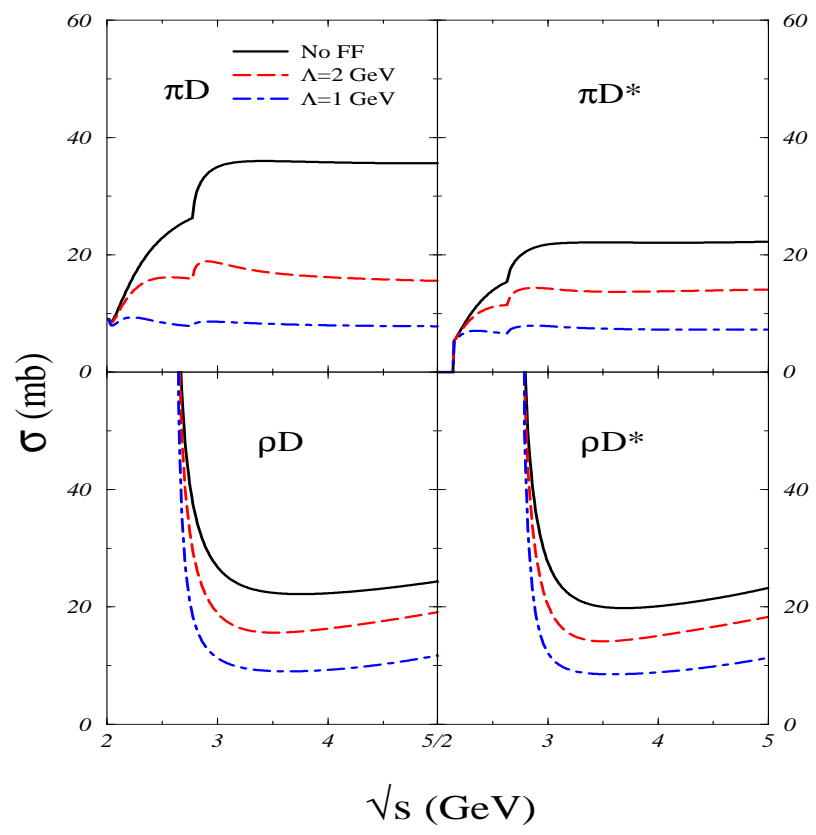

FIG. 2. Total cross section as functions of energy without and with form factors.

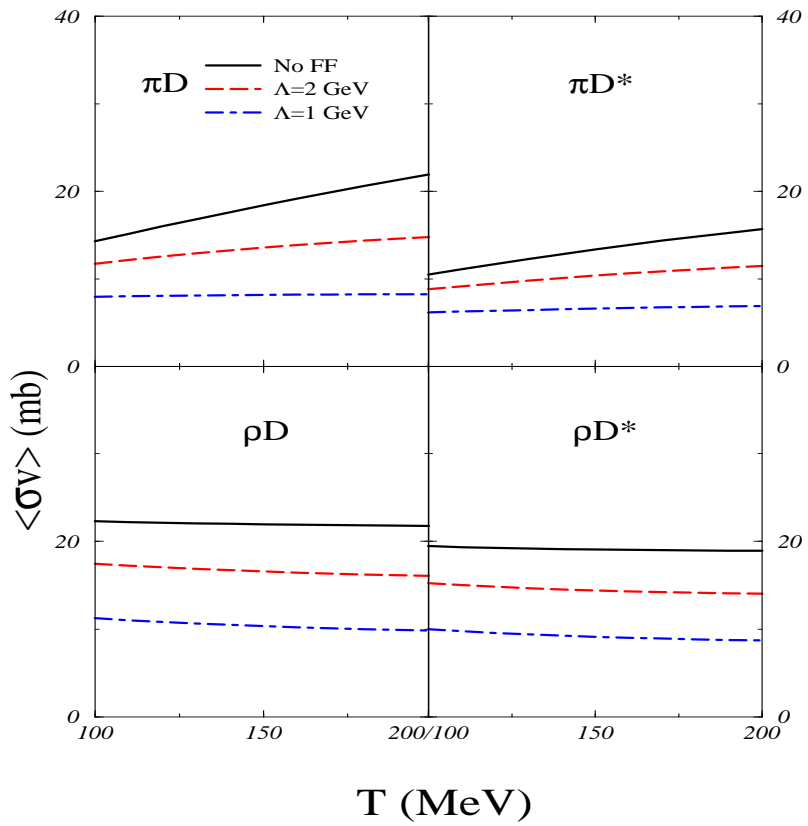

FIG. 3. Thermal average of the total cross section as functions of temperature without and with form factors. 
4 for $\pi D^{*}$ scattering, processes 5 and 6 for $\rho D$ scattering, and processes 7 and 8 for $\rho D^{*}$ scattering. We have also evaluated the thermal average of these cross sections, and they are shown by the solid lines in Fig. 3 as functions of temperature.

To study the effects due to form factors, we take the value for the cutoff parameter $\Lambda$ as either 2 or $1 \mathrm{GeV}$. The results are shown in Figs. 2 and 3 by the dashed and dash-dotted curves, respectively. As expected, magnitude of the cross sections decreases with decreasing cutoff parameter. For the cutoff parameters used here, the cross sections for $\pi D, \pi D^{*}, \rho D$ and $\rho D^{*}$ scatterings are all roughly between 10 and $20 \mathrm{mb}$. Compared to the case without form factors, we see that form factors only suppress modestly (by a factor of two of less) the total cross sections and their thermal averages. This is due to the dominance of elastic processes, which involve small momentum transfer near the threshold. In contrast, form factors suppress significantly the cross section for $J / \psi$ absorption by pion, e.g., the process $\pi \psi \rightarrow D^{*} \bar{D}$ is reduced by as much as a factor of 8 due to its large threshold [7].

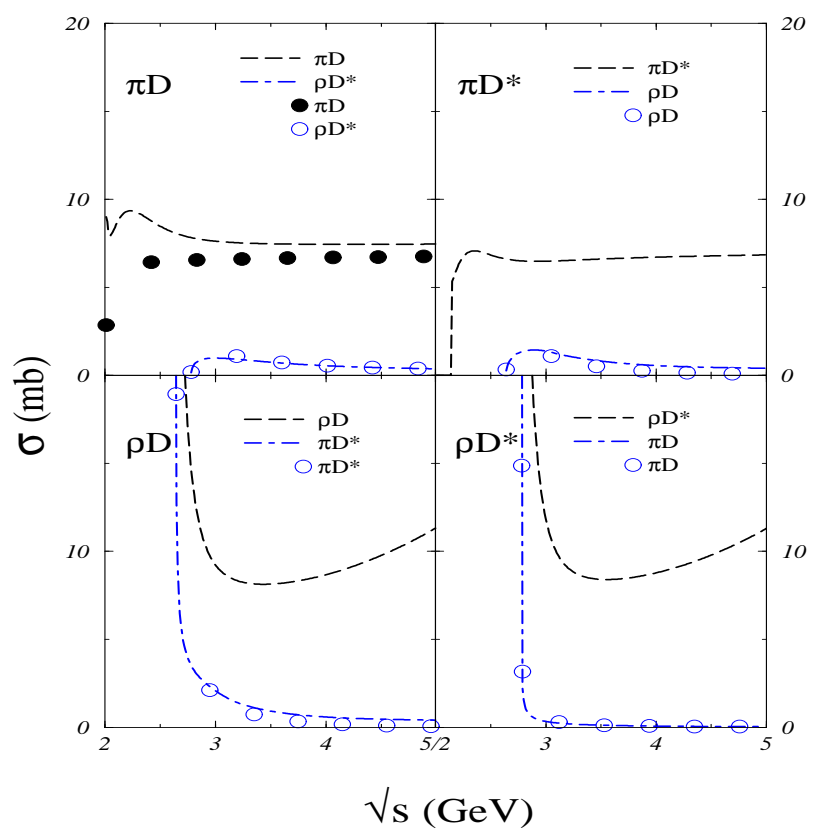

FIG. 4. Individual cross sections with form factors $(\Lambda=1 \mathrm{GeV})$ as functions of energy. Circles represent results based on the previous study that includes only the pseudoscalar-pseudoscalar-vector meson couplings and the light meson exchanges [3].

In Figs. 6 and 5, we show by the dashed and dot-dashed curves the cross sections for individual processes and their thermal averages for a cutoff parameter of $1 \mathrm{GeV}$. It is seen that cross sections for both processes $2\left(\pi D \rightarrow \rho D^{*}\right)$ and $4\left(\pi D^{*} \rightarrow \rho D\right)$ increase from zero at their respective threshold, while cross sections for processes $6\left(\rho D \rightarrow \pi D^{*}\right)$ and $8\left(\rho D^{*} \rightarrow \pi D\right)$ diverge near threshold because they are exothermic. For the four elastic processes $1,3,5$, and 7 , their cross sections are finite at threshold. We also note that elastic processes are much more important than corresponding inelastic processes.

Fig. [6 shows the thermal averages of cross sections for processes 1, 3, 4 and 6 at $T=150$ $\mathrm{MeV}$ as functions of the imaginary self-energy $\Gamma$ of the exchanged meson. It is seen that they do not vary much for values of $\Gamma$ between 5 and $500 \mathrm{MeV}$. 


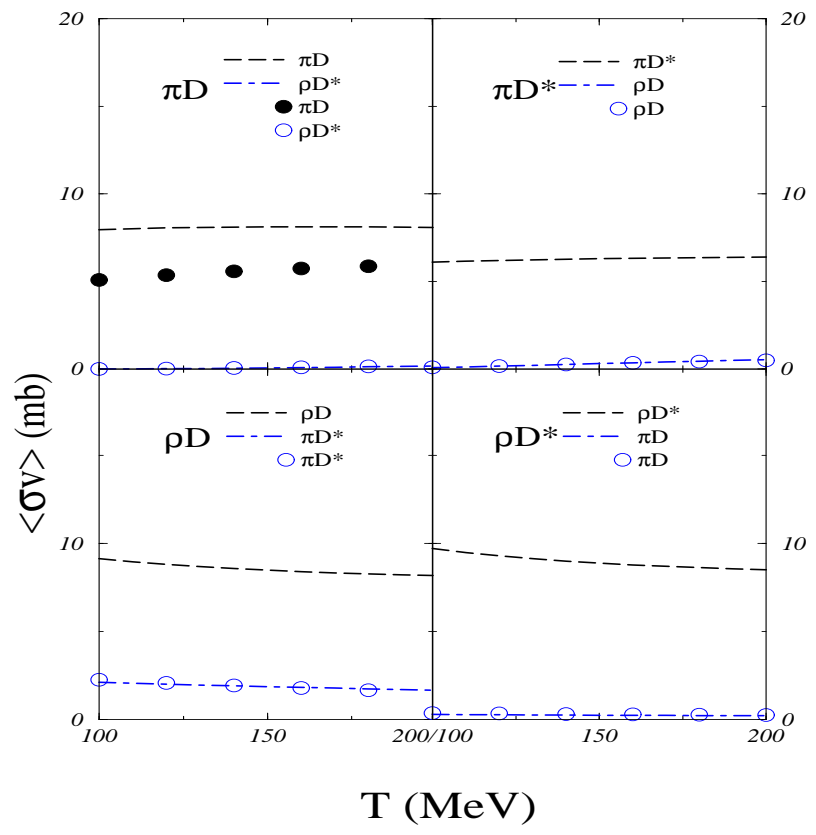

FIG. 5. Thermal averages of individual cross sections with form factors $(\Lambda=1 \mathrm{GeV})$ as functions of temperature. Circles represent results based on the previous study that includes only the pseudoscalar-pseudoscalar-vector meson couplings and the light meson exchanges [3].

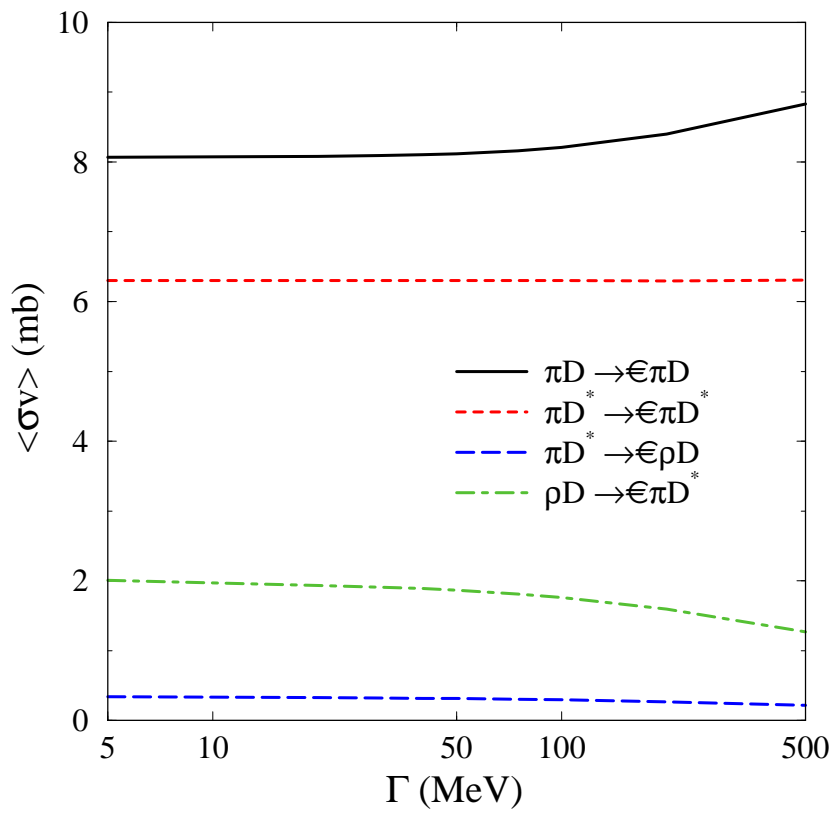

FIG. 6. Thermal average of cross sections at $T=150 \mathrm{MeV}$ with form factors $(\Lambda=1 \mathrm{GeV})$ as functions of the imaginary self-energy of the exchanged meson. 


\section{COMPARISON WITH PREVIOUS RESULTS}

In our previous study of charm meson scattering cross sections [3], we have considered only the pseudoscalar-pseudoscalar-vector meson interactions, which lead to diagrams 1a-c, 2a-b, 3b-c, 4a, 4c, 5b-c, 6a, 6c, and 8a-b in Fig. 1. Since diagrams involving charm mesons are expected to be less important, we have evaluated only those diagrams that involve the exchange of light mesons ( $\pi$ or $\rho$ meson), i.e., diagrams (a) in processes $1,2,4,6$, and 8 . These results are shown by circles in Figs. 1 and 5 . Except for process $1(\pi D \rightarrow \pi D)$ near the threshold, they are close to present results that include also charm meson exchanges and contact terms. This comparison thus demonstrates explicitly that diagrams with light meson exchanges dominate the charm meson scattering cross sections. This is in contrast to the charmonium absorption cross section in hadronic matter, where inclusion of additional couplings among three vector mesons and among four particles increases the $J / \psi$ absorption cross section by pion by an order of magnitude [6,,7]. This is due to the absence of light meson exchanges in charmonium absorption by hadrons.

We note that these additional interactions among three vector mesons and among four particles yield new processes with light meson exchanges, i.e., diagrams 3a, 5a, and 7a. We have checked that these diagrams also dominate the cross sections for these processes. As shown in Fig. 4 and 5, these elastic processes are more important than corresponding inelastic processes and thus increase significantly the total scattering cross sections of charm mesons by pion and rho meson.

\section{SUMMARY}

In summary, we have studied the scattering cross sections of charm mesons by pion and rho meson using a gauge invariant hadronic Lagrangian generated from the SU(4) symmetry. This leads to interaction Lagrangians not only among two pseudoscalar mesons and one vector meson but also among three vector mesons as well as among four particles. We have found that the charm meson scattering cross sections are dominated by diagrams with light meson exchanges. For the processes considered previously based only on the pseudoscalarpseudoscalar-vector meson interactions, these additional interaction Lagrangians do not introduce new diagrams with light meson exchanges, and their cross sections are thus not much affected. However, the interaction Lagrangians involving three vector mesons or four particles lead to new processes with light meson exchanges besides those considered previously and thus increase the total charm meson scattering cross sections by hadrons. Therefore, we expect as in the previous study that the charm meson spectra in heavy ion collisions can be significantly modified by hadronic scattering.

\section{ACKNOWLEDGMENTS}

This work was supported in part by the National Science Foundation under Grant No. PHY-9870038, the Welch Foundation under Grant No. A-1358, and the Texas Advanced Research Program under Grant Nos. FY97-010366-0068 and FY99-010366-0081. 


\section{APPENDIX A}

In this appendix, we give the explicit expressions for the amplitudes of all diagrams in Fig. 1. We show only the reduced amplitudes without the polarization vectors of external vector mesons and before summing and averaging over external spins and isospins.

For process $1, \pi D \rightarrow \pi D$, we have

$$
\begin{aligned}
& \mathcal{M}_{1 a}=g_{\rho \pi \pi} g_{\rho D D}\left(-\imath \epsilon_{i j k} \tau^{k}\right)_{\alpha \beta}\left(\frac{1}{t-m_{\rho}^{2}}\right)(s-u), \\
& \mathcal{M}_{1 b}=g_{\pi D D^{*}}^{2}\left(\tau^{j} \tau^{i}\right)_{\alpha \beta}\left(\frac{1}{u-m_{D^{*}}^{2}}\right)\left[s-t-\frac{\left(m_{D}^{2}-m_{\pi}^{2}\right)^{2}}{m_{D^{*}}^{2}}\right], \\
& \mathcal{M}_{1 c}=g_{\pi D D^{*}}^{2}\left(\tau^{i} \tau^{j}\right)_{\alpha \beta}\left(\frac{1}{s-m_{D^{*}}^{2}}\right)\left[-t+u-\frac{\left(m_{D}^{2}-m_{\pi}^{2}\right)^{2}}{m_{D^{*}}^{2}}\right] .
\end{aligned}
$$

For process $2, \pi D \rightarrow \rho D^{*}$, we have

$$
\begin{aligned}
\mathcal{M}_{2 a}^{\lambda \omega} & =g_{\rho \pi \pi} g_{\pi D D^{*}}\left(\imath \epsilon_{i j k} \tau^{k}\right)_{\alpha \beta}\left(-2 p_{1}+p_{3}\right)^{\lambda}\left(\frac{1}{t-m_{\pi}^{2}}\right)\left(p_{1}-p_{2}-p_{3}\right)^{\omega}, \\
\mathcal{M}_{2 b}^{\lambda \omega} & =g_{\pi D D^{*}} g_{\rho D D}\left(\tau^{j} \tau^{i}\right)_{\alpha \beta}\left(-p_{1}+p_{2}+p_{4}\right)^{\lambda}\left(\frac{1}{u-m_{D}^{2}}\right)\left(-2 p_{1}+p_{4}\right)_{\omega}, \\
\mathcal{M}_{2 c}^{\lambda \omega} & =g_{\pi D D^{*}} g_{\rho D^{*} D^{*}}\left(\tau^{i} \tau^{j}\right)_{\alpha \beta}\left(p_{1}-p_{2}\right)^{\gamma}\left(\frac{1}{s-m_{D^{*}}^{2}}\right)\left[g_{\gamma \gamma^{\prime}}-\frac{\left(p_{1}+p_{2}\right)_{\gamma}\left(p_{1}+p_{2}\right)_{\gamma^{\prime}}}{m_{D^{*}}^{2}}\right] \\
& \times\left[\left(-p_{1}-p_{2}-p_{4}\right)^{\lambda} g^{\gamma^{\prime} \omega}+\left(-p_{3}+p_{4}\right)^{\gamma^{\prime}} g^{\lambda \omega}+\left(p_{1}+p_{2}+p_{3}\right)^{\omega} g^{\gamma^{\prime} \lambda}\right], \\
\mathcal{M}_{2 d}^{\lambda \omega} & =g_{\pi \rho D D^{*}}\left(\tau^{i} \tau^{j}-2 \tau^{j} \tau^{i}\right)_{\alpha \beta} g^{\lambda \omega} .
\end{aligned}
$$

In the above, $p_{n}$ denotes the momentum of particle $n$. Our convention is such that particles 1 and 2 represent initial-state mesons while particles 3 and 4 represent final-state mesons on the left and right side of the diagrams shown in Fig. 1, respectively. For vector mesons, the indices $\mu, \nu, \lambda$, and $\omega$ denote the polarization components of external mesons while the indices $\gamma$ and $\gamma^{\prime}$ denote those of the exchanged meson. The indices $i$ and $j$ represent the isospin state of isospin-triplet mesons on the left of a diagram, while the indices $\alpha$ and $\beta$ represent those of isospin-doublet mesons on the right of a diagram. For the isospin-triplet meson in the propagator the index $k$ represents its isospin state.

The amplitudes for processes 4, 6 and 8 are related to above amplitudes for process 2 by the crossing symmetry. For process $4, \pi D^{*} \rightarrow \rho D$, we then have

$$
\mathcal{M}_{4 a}^{\nu \lambda}=\hat{\mathrm{T}}_{4} \mathcal{M}_{2 a}^{\lambda \omega}, \mathcal{M}_{4 b}^{\nu \lambda}=\hat{\mathrm{T}}_{4} \mathcal{M}_{2 c}^{\lambda \omega}, \mathcal{M}_{4 c}^{\nu \lambda}=\hat{\mathrm{T}}_{4} \mathcal{M}_{2 b}^{\lambda \omega}, \mathcal{M}_{4 d}^{\nu \lambda}=\hat{\mathrm{T}}_{4} \mathcal{M}_{2 d}^{\lambda \omega}
$$

where $\hat{\mathrm{T}}_{4}$ represents the replacement, $p_{2} \leftrightarrow-p_{4}, \nu \leftrightarrow \omega$, and $i \leftrightarrow j$. For process $6, \rho D \rightarrow$ $\pi D^{*}$, we have

$$
\mathcal{M}_{6 a}^{\mu \omega}=\hat{\mathrm{T}}_{6} \mathcal{M}_{2 a}^{\lambda \omega}, \mathcal{M}_{6 b}^{\mu \omega}=\hat{\mathrm{T}}_{6} \mathcal{M}_{2 c}^{\lambda \omega}, \mathcal{M}_{6 c}^{\mu \omega}=\hat{\mathrm{T}}_{6} \mathcal{M}_{2 b}^{\lambda \omega}, \mathcal{M}_{6 d}^{\mu \omega}=\hat{\mathrm{T}}_{6} \mathcal{M}_{2 d}^{\lambda \omega}
$$

where $\hat{\mathrm{T}}_{6}$ represents the replacement, $p_{1} \leftrightarrow-p_{3}, \mu \leftrightarrow \lambda$, and $i \leftrightarrow j$. For process $8, \rho D^{*} \rightarrow$ $\pi D$, we have 


$$
\mathcal{M}_{8 a}^{\mu \nu}=\hat{\mathrm{T}}_{8} \mathcal{M}_{2 a}^{\lambda \omega}, \mathcal{M}_{8 b}^{\mu \nu}=\hat{\mathrm{T}}_{8} \mathcal{M}_{2 b}^{\lambda \omega}, \mathcal{M}_{8 c}^{\mu \nu}=\hat{\mathrm{T}}_{8} \mathcal{M}_{2 c}^{\lambda \omega}, \mathcal{M}_{8 d}^{\mu \nu}=\hat{\mathrm{T}}_{8} \mathcal{M}_{2 d}^{\lambda \omega},
$$

where $\hat{\mathrm{T}}_{8}$ represents the replacement, $p_{1} \leftrightarrow-p_{3}, p_{2} \leftrightarrow-p_{4}, \mu \leftrightarrow \lambda$, and $\nu \leftrightarrow \omega$.

For process $3, \pi D^{*} \rightarrow \pi D^{*}$, we have

$$
\begin{aligned}
\mathcal{M}_{3 a}^{\nu \omega} & =g_{\rho \pi \pi} g_{\rho D^{*} D^{*}}\left(-\imath \epsilon_{i j k} \tau^{k}\right)_{\alpha \beta}\left(\frac{1}{t-m_{\rho}^{2}}\right) \\
& \times\left[(u-s) g^{\nu \omega}+4\left(p_{1}^{\nu} p_{3}^{\omega}-p_{3}^{\nu} p_{1}^{\omega}\right)+p_{2}^{\nu}\left(p_{1}+p_{3}\right)^{\omega}+\left(p_{1}+p_{3}\right)^{\nu} p_{4}^{\omega}\right] \\
\mathcal{M}_{3 b}^{\nu \omega} & =g_{\pi D D^{*}}^{2}\left(\tau^{j} \tau^{i}\right)_{\alpha \beta}\left(-p_{1}-p_{3}+p_{4}\right)^{\nu}\left(\frac{1}{u-m_{D}^{2}}\right)\left(2 p_{1}-p_{4}\right)^{\omega} \\
\mathcal{M}_{3 c}^{\nu \omega} & =g_{\pi D D^{*}}^{2}\left(\tau^{i} \tau^{j}\right)_{\alpha \beta}\left(2 p_{1}+p_{2}\right)^{\nu}\left(\frac{1}{s-m_{D}^{2}}\right)\left(-p_{1}-p_{2}-p_{3}\right)^{\omega} \\
\mathcal{M}_{3 d}^{\nu \omega} & =g_{\pi \pi D^{*} D^{*}} \delta_{i j} \delta_{\alpha \beta} g^{\nu \omega} .
\end{aligned}
$$

For process $5, \rho D \rightarrow \rho D$, we have

$$
\begin{aligned}
\mathcal{M}_{5 a}^{\mu \lambda} & =g_{\rho \rho \rho} g_{\rho D D}\left(-\imath \epsilon_{i j k} \tau^{k}\right)_{\alpha \beta}\left(\frac{1}{t-m_{\rho}^{2}}\right) \\
& \times\left[(u-s) g^{\mu \lambda}+4\left(p_{2}^{\mu} p_{4}^{\lambda}-p_{4}^{\mu} p_{2}^{\lambda}\right)+p_{1}^{\mu}\left(p_{2}+p_{4}\right)^{\lambda}+3\left(p_{2}+p_{4}\right)^{\mu} p_{3}^{\lambda}\right], \\
\mathcal{M}_{5 b}^{\mu \lambda} & =g_{\rho D D}^{2}\left(-\tau^{j} \tau^{i}\right)_{\alpha \beta}\left(-p_{1}+2 p_{4}\right)^{\mu}\left(\frac{1}{u-m_{D}^{2}}\right)\left(-p_{1}+p_{2}+p_{4}\right)^{\lambda}, \\
\mathcal{M}_{5 c}^{\mu \lambda} & =g_{\rho D D}^{2}\left(-\tau^{i} \tau^{j}\right)_{\alpha \beta}\left(p_{1}+2 p_{2}\right)^{\mu}\left(\frac{1}{s-m_{D}^{2}}\right)\left(p_{1}+p_{2}+p_{4}\right)^{\lambda}, \\
\mathcal{M}_{5 d}^{\mu \lambda} & =g_{\rho \rho D D} \delta_{i j} \delta_{\alpha \beta} g^{\mu \lambda} .
\end{aligned}
$$

For process $7, \rho D^{*} \rightarrow \rho D^{*}$, we have

$$
\begin{aligned}
\mathcal{M}_{7 a}^{\mu \nu \lambda \omega} & =g_{\rho \rho \rho} g_{\rho D^{*} D^{*}}\left(\imath \epsilon_{i j k} \tau^{k}\right)_{\alpha \beta}\left(\frac{1}{t-m_{\rho}^{2}}\right) \\
& \times\left[\left(p_{1}+p_{3}\right)^{\gamma} g^{\mu \lambda}+\left(p_{1}-2 p_{3}\right)^{\mu} g^{\gamma \lambda}+\left(-2 p_{1}+p_{3}\right)^{\lambda} g^{\mu \gamma}\right] g_{\gamma \gamma^{\prime}} \\
& \times\left[\left(-p_{2}-p_{4}\right)^{\gamma^{\prime}} g^{\nu \omega}+\left(-p_{1}+p_{2}+p_{3}\right)^{\omega} g^{\gamma^{\prime} \nu}+\left(p_{1}-p_{3}+p_{4}\right)^{\nu} g^{\gamma^{\prime} \omega}\right], \\
\mathcal{M}_{7 b}^{\mu \nu \lambda \omega} & =g_{\rho D^{*} D^{*}}^{2}\left(\tau^{j} \tau^{i}\right)_{\alpha \beta}\left(\frac{1}{u-m_{D^{*}}^{2}}\right) \\
& \times\left[\left(p_{1}+p_{4}\right)^{\gamma} g^{\mu \omega}+\left(p_{1}-2 p_{4}\right)^{\mu} g^{\gamma \omega}+\left(-2 p_{1}+p_{4}\right)^{\omega} g^{\gamma \mu}\right]\left[g_{\gamma \gamma^{\prime}}-\frac{\left(p_{1}-p_{4}\right)_{\gamma}\left(p_{1}-p_{4}\right)_{\gamma^{\prime}}}{m_{D^{*}}^{2}}\right] \\
& \times\left[\left(p_{1}+p_{3}-p_{4}\right)^{\nu} g^{\gamma^{\prime} \lambda}-\left(p_{2}+p_{3}\right)^{\gamma^{\prime}} g^{\nu \lambda}+\left(-p_{1}+p_{2}+p_{4}\right)^{\lambda} g^{\gamma^{\prime} \nu}\right], \\
\mathcal{M}_{7 c}^{\mu \nu \lambda \omega} & =g_{\rho D^{*} D^{*}}^{2}\left(\tau^{i} \tau^{j}\right)_{\alpha \beta}\left(\frac{1}{s-m_{D^{*}}^{2}}\right) \\
& \times\left[\left(2 p_{1}+p_{2}\right)^{\nu} g^{\gamma \mu}-\left(p_{1}+2 p_{2}\right)^{\mu} g^{\nu \gamma}+\left(-p_{1}+p_{2}\right)^{\gamma} g^{\mu \nu}\right]\left[g_{\gamma \gamma^{\prime}}-\frac{\left(p_{1}+p_{2}\right)_{\gamma}\left(p_{1}+p_{2}\right)_{\gamma^{\prime}}}{m_{D^{*}}^{2}}\right] \\
& \times\left[\left(-p_{3}+p_{4}\right)^{\gamma^{\prime}} g^{\lambda \omega}+\left(-p_{1}-p_{2}-p_{4}\right)^{\lambda} g^{\gamma^{\prime} \omega}+\left(p_{1}+p_{2}+p_{3}\right)^{\omega} g^{\gamma^{\prime} \lambda}\right], \\
\mathcal{M}_{7 d}^{\mu \nu \lambda \omega} & =g_{\rho \rho D^{*} D^{*}}\left[\left(2 \tau^{j} \tau^{i}-\tau^{i} \tau^{j}\right)_{\alpha \beta} g^{\mu \nu} g^{\lambda \omega}+\left(2 \tau^{i} \tau^{j}-\tau^{j} \tau^{i}\right)_{\alpha \beta} g^{\mu \omega} g^{\nu \lambda}-2 \delta_{i j} \delta_{\alpha \beta} g^{\mu \lambda} g^{\nu \omega}\right] .(\mathrm{A} 8)
\end{aligned}
$$


After averaging (summing) over initial (final) spins and isopsins, the cross section for a process is given by

$$
\frac{d \sigma_{n}}{d t}=\frac{1}{64 \pi s p_{i, \mathrm{~cm}}^{2} I_{s} I_{i}} \mathcal{M}_{n}^{\lambda_{k} \cdots \lambda_{l}} \mathcal{M}_{n}^{* \lambda_{k}^{\prime} \cdots \lambda_{l}^{\prime}}\left(g_{\lambda_{k} \lambda_{k}^{\prime}}-\frac{p_{k \lambda_{k}} p_{k \lambda_{k}^{\prime}}}{m_{k}^{2}}\right) \cdots\left(g_{\lambda_{l} \lambda_{l}^{\prime}}-\frac{p_{l \lambda_{l}} p_{l \lambda_{l}^{\prime}}}{m_{l}^{2}}\right),
$$

with $s, t, u$ being the standard Mandelstam variables, and

$$
p_{i, \mathrm{~cm}}^{2}=\frac{\left[s-\left(m_{1}+m_{2}\right)^{2}\right]\left[s-\left(m_{1}-m_{2}\right)^{2}\right]}{4 s}
$$

is the squared momentum of initial-state mesons in the center-of-momentum frame. The factors $I_{s}$ and $I_{i}$ are due to averaging over initial spin and isospins, respectively. Values of $I_{s}$ are $1,1,3,3,3,3,9$ and 9 , respectively, for processes 1 to 8 in Fig. 1, while $I_{i}$ is 6 for all processes.

\section{APPENDIX B}

In this appendix, we show as an example that the scattering amplitude for process 2, $\pi D \rightarrow \rho D^{*}$, satisfies the condition of Eq. (8) as a result of the $\mathrm{SU}(4)$ flavor current conservation. In particular, we shall prove that $\mathcal{M}_{2}^{\lambda \omega} p_{4 \omega}=0$. Starting from Eq. (A2), we obtain

$$
\begin{aligned}
\mathcal{M}_{2 a}^{\lambda \omega} p_{4 \omega} & =g_{\rho \pi \pi} g_{\pi D D^{*}}\left(\imath \epsilon_{i j k} \tau^{k}\right)_{\alpha \beta}\left(\frac{t-m_{D}^{2}}{t-m_{\pi}^{2}}\right)\left(-2 p_{1}\right)^{\lambda}, \\
\mathcal{M}_{2 b}^{\lambda \omega} p_{4 \omega} & =g_{\pi D D^{*}} g_{\rho D D}\left(\tau^{j} \tau^{i}\right)_{\alpha \beta}\left(\frac{u-m_{\pi}^{2}}{u-m_{D}^{2}}\right)\left(-p_{1}+p_{2}+p_{4}\right)^{\lambda}, \\
\mathcal{M}_{2 c}^{\lambda \omega} p_{4 \omega} & =g_{\pi D D^{*}} g_{\rho D^{*} D^{*}}\left(\tau^{i} \tau^{j}\right)_{\alpha \beta}\left(\frac{1}{s-m_{D^{*}}^{2}}\right)\left[\left(s-m_{\rho}^{2}\right)\left(p_{1}-p_{2}\right)^{\lambda}\right. \\
& \left.+\frac{\left(m_{D}^{2}-m_{\pi}^{2}\right)\left(m_{D^{*}}^{2}-m_{\rho}^{2}\right)}{m_{D^{*}}^{2}} p_{4}^{\lambda}\right], \\
\mathcal{M}_{2 d}^{\lambda \omega} p_{4 \omega} & =g_{\pi \rho D D^{*}}\left(\tau^{i} \tau^{j}-2 \tau^{j} \tau^{i}\right)_{\alpha \beta} p_{4}^{\lambda} .
\end{aligned}
$$

In arriving at the above, we have discarded all terms with $p_{3}^{\lambda}$ as they vanish after contracting with the polarization vector $\epsilon_{3 \lambda}$. Using the $\mathrm{SU}(4)$ relation for the coupling constants shown in Eq. (10) then gives

$$
\begin{aligned}
\mathcal{M}_{2}^{\lambda \omega} p_{4 \omega} & =\frac{g^{2}}{8}\left(\tau^{j} \tau^{i}\right)_{\alpha \beta}\left(m_{D}^{2}-m_{\pi}^{2}\right)\left(\frac{-p_{1}^{\lambda}}{t-m_{\pi}^{2}}+\frac{p_{2}^{\lambda}}{u-m_{D}^{2}}\right) \\
& +\frac{g^{2}}{16}\left(\tau^{i} \tau^{j}\right)_{\alpha \beta}\left[2\left(\frac{m_{D}^{2}-m_{\pi}^{2}}{t-m_{\pi}^{2}}\right) p_{1}^{\lambda}+\left(\frac{m_{D^{*}}^{2}-m_{\rho}^{2}}{s-m_{D^{*}}^{2}}\right)\left(p_{1}-p_{2}\right)^{\lambda}\right. \\
& \left.+\frac{\left(m_{D}^{2}-m_{\pi}^{2}\right)\left(m_{D^{*}}^{2}-m_{\rho}^{2}\right)}{m_{D^{*}}^{2}\left(s-m_{D^{*}}^{2}\right)}\left(p_{1}+p_{2}\right)^{\lambda}\right]
\end{aligned}
$$

With degenerate pseudoscalar meson masses and degenerate vector meson masses, the above expression then reduces to zero. For other amplitudes shown in Eqs. (A3)-(A8), the current conservation condition can be similarly proved. We note that $\mathcal{M}_{2}^{\lambda \omega} p_{3 \lambda}=0$ holds for any masses. 


\section{REFERENCES}

[1] E. Shuryak, Phys. Rev. C 55, 961 (1997).

[2] Z. Lin, R. Vogt, and X.-N. Wang, Phys. Rev. C 57, 899 (1998).

[3] Z. Lin, C. M. Ko, and B. Zhang, Phys. Rev. C 61, 024904 (2000).

[4] Z. Lin and X.-N. Wang, Phys. Lett. B 444, 245 (1998).

[5] S. G. Matinyan and B. Müller, Phys. Rev. C 58, 2994 (1998).

[6] K. Haglin, Phys. Rev. C 61, 031902 (2000).

[7] Z. Lin and C. M. Ko, Phys. Rev. C 62, 034903 (2000).

[8] G. Q. Li and C. M. Ko, Nucl. Phys. A 594, 439 (1995); W. Peters, U. Mosel, and A. Engel, Z. Phys. A 353, 333 (1996); R. Baier, M. Dirks, and K. Redlich, Phys. Rev. D 55, 4344 (1997).

[9] P. Colangelo, F. De Fazio, and G. Nardulli, Phys. Lett. B 334, 175 (1994); V. M. Belyaev, V. M. Braun, A. Khodjamirian, and R. Ruckl, Phys. Rev. D 51, 6177 (1995).

[10] G. E. Brown, C. M. Ko, Z. G. Wu and L. H. Xia, Phys. Rev. C 43, 1881 (1991); C. M. Ko and D. Seibert, Phys. Rev. C 49, 2198 (1994).

[11] K. Tsushima, S. W. Huang, and A. Faessler, Phys. Lett. B 337, 245 (1994); E. L. Bratkovskaya and C. M. Ko, Phys. Lett. B 445, 265 (1999).

[12] J. Kapusta, P. Lichard, and D. Seibert, Phys. Rev. D 44, 2774 (1991); 47, 4171 (E) (1993).

[13] K. Haglin and C. Gale, nucl-th/0010017.

[14] We have realized that there is a typo in the expression for the thermal averaged cross section in Ref. [3]. The factors $\alpha_{1}$ and $\alpha_{2}$ in the denominator outside the Bessel functions should be $\alpha_{1}^{2}$ and $\alpha_{2}^{2}$, respectively. 